\title{
APLICACIÓN ACADÉMICA DE MENSAJES DE TEXTO EN UN CURSO DE PRIMEROS AUXILIOS: ESTUDIO PILOTO EN UNA UNIVERSIDAD PRIVADA DE LIMA, PERÚ
}

\author{
Rodrigo M. Carrillo-Larco1,2,a, Sebastián B. Shu-Yip ${ }^{1,2, a}$, José E. Pérez-Lu³,b
}

RESUMEN

El objetivo del estudio fue desarrollar y evaluar el impacto de una intervención educativa con el uso de mensajes de texto (SMS) en un curso de primeros auxilios. Se realizó un estudio prospectivo y de intervención. Se implementaron dos etapas: 1) estudio cualitativo para diseñar los SMS, 2) intervención con el envío de los SMS. La variable resultado fue la nota final de los alumnos. Se construyeron modelos multivariados y se calculó razones de prevalencia e intervalos de confianza al 95\%. La primera fase reveló que los SMS debían ser educativos-teóricos, motivacionales, y tipo test. En la intervención hubo 66 participantes en el grupo control e intervención. La edad promedio fue $17,7( \pm 1,2)$ años. El grupo intervención obtuvo calificación mayor en comparación con el grupo control (RP=4,82; IC 95\%: 1,58-14,72). En conclusión, los SMS con contenido informativo y motivacional son útiles en la formación del pregrado en Medicina.

Palabras clave: Educación médica; Tecnología educativa; Tecnología biomédica (fuente: DeCS BIREME).

\section{ACADEMIC APPLICATION OF TEXT MESSAGES IN A FIRST AID COURSE: A PILOT STUDY IN A PRIVATE UNIVERSITY IN LIMA, PERU}

\begin{abstract}
The aim of the study was to develop and evaluate the impact of an educational intervention with the use of text messages (SMS) in a first aid course. A prospective study and intervention was conducted. Two steps were implemented: 1) a qualitative study to design the SMS and 2) the intervention with the sending of the SMS messages. The outcome variable was the final grade of students. Multivariate models were constructed, prevalence ratios and confidence intervals at $95 \%$ were calculated. The first phase revealed that the SMS should be educational-theoretical, motivational, and multiple choice. In the intervention there were 66 participants in the control and intervention group. The average age was $17.7( \pm 1.2)$ years. The intervention group obtained higher scores compared with the control group ( $\mathrm{PR}=4.82 ; 95 \% \mathrm{Cl}$ : 1.58 to 14.72$)$. In conclusion, SMS with informative and motivational content is useful in the formation of undergraduate medicine.
\end{abstract}

Key words: Education, medical; Educational technology; Biomedical technology (source: MeSH NLM).

\section{INTRODUCCIÓN}

Actualmente Twitter y Facebook son empleados para la promoción de la investigación y para mejorar la comunicación durante conferencias en salud $(1,2)$. Asimismo, archivos multimedia (ej. podcast) son utilizados por estudiantes como herramientas académicas ${ }^{(3)}$. Sin embargo, estas tecnologías requieren de conexión a Internet, lo cual no siempre se encuentra disponible. Es así que los mensajes de texto (SMS) son una opción útil ya que solo requieren de un celular básico. Instituciones en el campo de la salud y facultades de medicina han incluido a los SMS en varios programas de entrenamiento ${ }^{(4,5,6)}$. A pesar de ello, se necesita generar más evidencia sobre el aprendizaje electrónico ${ }^{(6,7)}$, y aun más sobre el uso de SMS en la educación médica en estudiantes de pregrado de Medicina.

El objetivo del estudio fue desarrollar y evaluar el impacto de una intervención educativa con el uso de SMS en estudiantes de medicina de una universidad privada en Lima, Perú.

Facultad de Medicina "Alberto Hurtado", Universidad Peruana Cayetano Heredia, Lima, Perú.

Sociedad Científica de Estudiantes de Medicina Cayetano Heredia (SOCEMCH), Facultad de Medicina "Alberto Hurtado", Universidad Peruana Cayetano Heredia, Lima, Perú.

Facultad de Salud Pública y Administración, Universidad Peruana Cayetano Heredia, Lima, Perú.

Estudiante de Medicina; ${ }^{\mathrm{b}}$ Médico cirujano

Recibido: : 18-02-15 Aprobado: 13-05-15

Citar como: Carrillo-Larco RM, Shu-Yip SB, Pérez-Lu JE. Aplicación académica de mensajes de texto en un curso de primeros auxilios: estudio piloto en una universidad privada de Lima, Perú. Rev Peru Med Exp Salud Publica. 2015;32(2):278-82. 


\section{EL ESTUDIO}

\section{DISEÑO}

Estudio prospectivo y de intervención, realizado en dos fases: la primera incluyó un estudio cualitativo con el fin de recopilar información para diseñar la intervención (segunda fase). La primera fase corresponde al objetivo de diseñar, y la segunda al objetivo de evaluar la intervención.

\section{ESCENARIO}

La investigación se realizó el año 2013, en la Universidad Peruana Cayetano Heredia (UPCH) en Lima, Perú. El estudio se enfocó al curso de Primeros Auxilios, que es una asignatura preclínica dirigida a los alumnos del primer año de la Facultad Medicina. La evaluación del curso incluía promedio de evaluación en clase (ej. evaluaciones semanales) que era equivalente al $30 \%$ de la calificación final, práctica en servicios hospitalarios equivalente al $10 \%$, examen teórico final equivalente al $20 \%$, y examen práctico final equivalente al $40 \%$. Cabe mencionar que tres de estos rubros incluían en el método de evaluación un componente cognitivo.

\section{PARTICIPANTES}

Los participantes de la primera fase fueron estudiantes de Medicina del segundo año, quienes completaron el curso de Primeros Auxilios en el año 2012. Se realizaron dos grupos focales, cada uno de siete alumnos. Los invitados fueron seleccionados para proveer información sobre qué temas fueron los más complejos, qué técnicas de estudio utilizaron, qué temas consideraron los más importantes, y qué tipo de SMS les hubiera gustado recibir si ellos hubiesen recibido esta intervención. Esta metodología fue empleada para diseñar los mensajes que se enviaron en la segunda fase.

Para la segunda fase, toda la población de estudiantes del primer año de Medicina (clase 2013) fue invitada a participar del estudio. Los alumnos del primer año se dividieron en cuatro grupos. Cada grupo contaba con cuatro clases teóricas-prácticas del curso Primeros Auxilios. Cada grupo tomó el curso cuando el grupo previo lo había terminado. Esta metodología permitió tener grupos control y de intervención evitando el riesgo de contaminación. Los dos primeros grupos fueron control y los dos últimos, intervención. Consideramos que si los dos primeros grupos hubiesen recibido la intervención ellos hubieran podido compartir la información con sus pares quienes recibirían el curso posteriormente.

\section{JUSTIFICACIÓN DE LA INTERVENCIÓN}

La capacidad de los SMS en establecer cortas revisiones y breves evaluaciones periódicamente va acorde con la teoría Space Study. Se ha descrito que cuando a los estudiantes se les da información sobre un tema específico dividida en dos sesiones separadas por un intervalo de tiempo, se obtiene mejores resultados que si reciben la información en una sola sesión ${ }^{(8)}$.

En el presente estudio, los participantes atendieron a sus clases regulares sobre un tema particular y una semana después recibieron un SMS con información relevante al tema de la semana previa.

\section{La intervención: primera fase}

Las sesiones de grupos focales fueron grabadas y transcritas. La información se analizó y se agrupó por temas. Los grupos focales fueron dirigidos por uno de los investigadores. Estas reuniones se realizaron en febrero de 2013.

\section{La intervención: segunda fase}

La intervención tuvo lugar durante el primer ciclo académico del año 2013. El grupo control fue reclutado entre el 5 y 12 de marzo; mientras que la intervención comenzó el 6 de mayo y duró hasta el 17 de junio. El primer SMS se enviaba los lunes al mediodía $(12: 00 \mathrm{~h})$, aquel mismo lunes a las 18:00 $\mathrm{h}$ se enviaba el complemento del primer mensaje y minutos después se enviaba el tercer mensaje de la semana. El cuarto, y último mensaje de la semana era enviado los jueves a las 18:00 h. En general, cada participante de la intervención recibió cuatro SMS cada semana. El contenido de los SMS fue escrito en castellano, contenían un máximo de 140 caracteres, y fueron enviados de forman manual por dos de los investigadores. En total se envió 24 mensajes a cada participante. A lo largo de la intervención la recepción de los SMS fue corroborada con los delegados de grupo, los cuales son personas elegidas por los propios estudiantes al inicio del ciclo académico.

\section{VARIABLES}

La variable resultado del estudio fue la calificación final del curso de Primero Auxilios que cada participante obtuvo, la calificación va de 0 a 20 . Para el análisis. la variable resultado fue dicotomizada: $\geq 18$ y $<18$ puntos (categoría de referencia en los modelos de regresión). Se decidió por 18 como punto de corte pues en varios patrones de equivalencia internacional 18 o más puntos equivale a "A" (excelente). La variable exposición fue haber recibido la intervención o no. 
Todos los participantes fueron encuestados para obtener su información sociodemográfica: sexo (masculino y femenino); edad (<18 y $\geq 18$ años); departamento de nacimiento (Lima o no de Lima); familiares cercanos que sean médicos de profesión (sí o no). Adicionalmente, se incluyó la variable modalidad de ingreso por la que el participante ingresó a la facultad de Medicina: examen general, alto rendimiento durante la secundaria, academia preuniversitaria (al menos un semestre) y otros (ej. bachillerato internacional).

\section{ANÁLISIS ESTADÍSTICO}

El análisis estadístico fue elaborado en STATA versión 11.0 (StataCorp; College Station, TX). Se calculó la proporción de cada variable categórica, también la media \pm desviación estándar para variables numéricas. Para determinar diferencias entre las características de ambos grupos se usó la prueba chi cuadrado para variables categóricas. Se utilizó un modelo lineal generalizado de la familia poisson ajustado por errores estándares robustos para calcular razones de prevalencia (RP) e intervalos de confianza al 95\% (IC 95\%).

\section{CONSIDERACIONES ÉTICAS}

El estudio fue aprobado por el Comité de Ética de la UPCH. Los participantes de la primera y segunda fase firmaron un consentimiento informado previo a su participación.

\section{HALLAZGOS}

El análisis de los grupos focales mostró que la intervención se beneficiaría con el uso de mensajes cuyo contenido encajara en tres categorías: a). Mensajes educativosteóricos; b). Mensajes motivacionales; c). Mensajes tipo test.

Los grupos focales revelaron que la manera más eficaz de programar el envío de los SMS sería: el primero de la semana sería un test para calcular la escala de coma de Glasgow; el segundo mensaje era la respuesta al primer mensaje; el tercer mensaje tenía contenido académico sobre un tema en particular, por ejemplo valores de temperatura para considerar fiebre según donde fuera medida (boca, axila, o rectal); finalmente, el cuarto mensaje buscaría motivar a los participantes a estudiar. En la Tabla 1 se presentan ejemplos de los SMS.

La elección de los días y horas para el envío de los SMS fue resultado de los grupos focales. Se escogió lunes pues era un día antes a la nueva clase teórica de Primeros Auxilios en la que, además, se tomaría una
Tabla 1. Ejemplo de los mensajes de texto

\begin{tabular}{ll}
\hline \multicolumn{1}{c}{ Tipo de SMS } & \multicolumn{1}{c}{ Ejemplo } \\
\hline Escenario & Caso: un paciente responde con un gruñido y \\
hipotético: & $\begin{array}{l}\text { retira el estímulo doloroso. Durante todo este } \\
\text { tiempo el paciente no abre los ojos ¿cuánto } \\
\text { escala de } \\
\text { Glasgow }\end{array}$ \\
$\begin{array}{l}\text { tiene de Glasgow? } \\
\text { Contenido }\end{array}$ & $\begin{array}{l}\text { VERBL: responde 5, confundido 4, palabras } \\
\text { inapropiadas 3, murmullos 2 y no responde 1. } \\
\text { OCULAR: espontaneo 4, estímulo verbal 3, } \\
\text { estímulo doloroso 2 y no responde 1. }\end{array}$ \\
& $\begin{array}{l}\text { Saber de primeros auxilios te da el poder de } \\
\text { salvar vidas iestudia, repasa y practica para } \\
\text { que algún día logres salvar muchas! }\end{array}$ \\
\hline
\end{tabular}

prueba sobre el contenido de la clase previa; de esta manera los mensajes del lunes permitían, recordaban y motivaban a estudiar. El jueves se escogió por ser considerado el día con menor carga académica de la semana, por lo tanto, un mensaje motivacional en este día se consideró oportuno para incentivar el estudio.

La muestra final fue 132 participantes (Figura 1). La edad media fue $17,7( \pm 1,2)$ años. La mayoría nació en Lima $(68,2 \%)$ y tenían un pariente médico $(55,3 \%)$; los detalles de las características de la población están especificados en la Tabla 2.

En general, la nota promedio fue 17,1 , la nota menor fue 14,6 y la máxima 18,7. Al dicotomizar la calificación final se evidenció que había mayor prevalencia de calificación superior (nota final >18) si los participantes habían recibido la intervención, en comparación a sus pares quienes no recibieron los SMS: RP: 4,82 (IC 95\%:

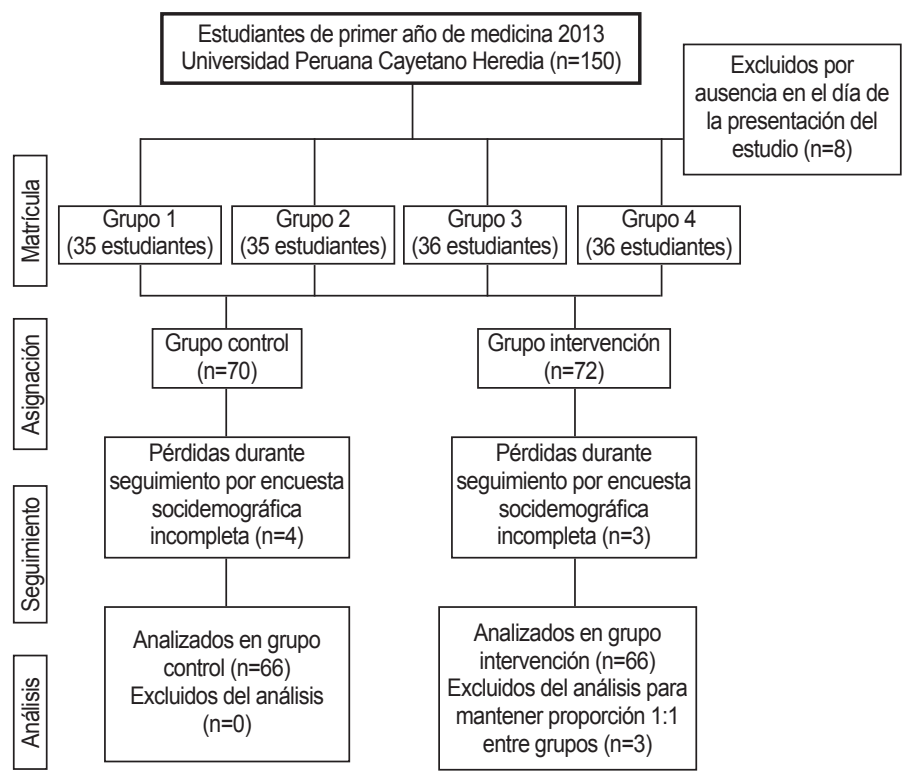

Figura 1. Flujograma de la población de estudio. 
Tabla 2. Características de la población de estudio por grupo control e intervención

\begin{tabular}{|c|c|c|c|}
\hline \multirow[t]{2}{*}{ Variables } & $\begin{array}{c}\text { Control } \\
(\%)\end{array}$ & $\begin{array}{c}\text { Intervención } \\
(\%)\end{array}$ & \multirow[t]{2}{*}{$p$} \\
\hline & $n=66$ & $\mathrm{~N}=66$ & \\
\hline \multicolumn{4}{|l|}{ Calificación final } \\
\hline$<18$ & 95,5 & 81,8 & 0,014 \\
\hline$\geq 18$ & 4,6 & 18,2 & \\
\hline \multicolumn{4}{|l|}{ Sexo } \\
\hline Masculino & 59,1 & 57,6 & 0,860 \\
\hline Femenino & 40,9 & 42,4 & \\
\hline \multicolumn{4}{|l|}{ Edad } \\
\hline$<18$ & 40,9 & 43,9 & 0,725 \\
\hline$\geq 18$ & 59,1 & 56,1 & \\
\hline \multicolumn{4}{|l|}{ Departamento de nacimiento } \\
\hline Lima & 63,6 & 72,7 & 0,262 \\
\hline No Lima & 36,4 & 27,3 & \\
\hline \multicolumn{4}{|c|}{ Modalidad de ingreso a la universidad } \\
\hline Examen general & 18,2 & 13,6 & 0,914 \\
\hline Ciclo preuniversitario & 31,8 & 33,3 & \\
\hline $\begin{array}{l}\text { Alto rendimiento durante } \\
\text { la secundaria }\end{array}$ & 34,9 & 36,4 & \\
\hline Otros & 15,2 & 16,2 & \\
\hline \multicolumn{4}{|l|}{ Parientes médicos } \\
\hline No & 40,9 & 48,5 & 0,381 \\
\hline Sí & 59,1 & 51,5 & \\
\hline
\end{tabular}

1,58-14,72). Los detalles del modelo multivariado se presentan en la Tabla 3.

\section{DISCUSIÓN}

El uso de SMS como herramienta didáctica en la formación del pregrado de medicina debiera incluir mensajes informativos y motivacionales. El grupo de intervención obtuvo en promedio mejor calificación que aquel sin la intervención.

Estos resultados son similares a otras experiencias en pregrado y posgrado ${ }^{(9-13)}$. Asimismo, un estudio en un

Tabla 3. Razón de prevalencia de obtener calificación $\geq 18$ por grupo control e intervención

\begin{tabular}{lcccc}
\hline \multirow{2}{*}{ Variable } & \multicolumn{2}{c}{ No ajustado } & \multicolumn{2}{c}{ Ajustado* } \\
\cline { 2 - 5 } & RP & $\mathbf{9 5 \% I C}$ & RP & $\mathbf{9 5 \% I C ~}$ \\
\hline Grupo & & & & \\
Control $(n=66)$ & 1,00 & & 1,00 & \\
$\begin{array}{l}\text { Intervención } \\
(n=66)\end{array}$ & 4,00 & $1,18-13,59$ & 4,82 & $1,58-14,72$ \\
\hline
\end{tabular}

*Ajustado por sexo, edad, departamento de nacimiento del participante, pariente médico y modalidad de ingreso del participante a la faculta de Medicina curso de Dermatología obtuvo conclusiones similares al presente estudio ${ }^{(14)}$. Sin embargo, su muestra estuvo compuesta por alumnos de un nivel superior. Por lo tanto, parece ser que el impacto positivo de los SMS en la educación médica se extiende a diferentes niveles dentro de la formación médica.

La muestra en este estudio estuvo compuesta por estudiantes del primer año de Medicina, muchos de los cuales acabaron la educación secundaria el año anterior, y se encuentran en un periodo de adaptación al ritmo universitario. Por lo tanto, es probable que se tenga mejor impacto en alumnos de años superiores, quienes pueden tener más herramientas de estudio, más conocimiento, y están mejor adaptados a las exigencias académicas.

Por otro lado, el ingreso a la formación médica es distinto según el sistema educativo de cada país. Por ejemplo, en el sistema peruano se ingresa a la escuela de Medicina después de haber acabado la educación secundaria. En otros países es necesario hacer primero un bachillerato. Por ende, es probable que los resultados, y así el efecto de los SMS en la educación médica, sea diferente según el sistema educativo o los requisitos que cada alumno deba cumplir para estar en una escuela de Medicina. Esto puede limitar la comparación entre estudios en distintos países.

El curso escogido para la intervención mostró escasa variabilidad de calificaciones, esto pudo haber evitado encontrar el máximo efecto de la intervención. Vale decir, si el uso de los SMS es útil para mejorar un rendimiento de por sí alto, o es más útil para llevar un bajo rendimiento a uno promedio o alto. Se recomienda realizar otras intervenciones que incluyan cursos con mayor variabilidad en sus calificaciones. Asimismo, se sugiere realizar otros estudios con más participantes, mayor tiempo de intervención, y dirigido a otros años de la educación médica.

Los resultados obtenidos y los reportados por otros autores muestran que los SMS son útiles para mejorar el entrenamiento y la educación de futuros médicos. Sin embargo, los médicos no son los únicos en el campo de la salud, las enfermeras también podrían verse beneficiadas con este tipo de programas, lo cual ya ha sido probado ${ }^{(15)}$. De igual manera, otros profesionales de la salud podrían encontrar útil los SMS para fines de entrenamiento. Los SMS parecen ser útiles en la formación de profesiones de la salud, y pueden ser particularmente útiles en lugares en donde medios de comunicación más sofisticados no estén ampliamente disponibles. 
Una fortaleza del estudio es la metodología para el desarrollo del contenido y envío de los SMS: se incluyó la perspectiva de los estudiantes (podría aumentar el interés de leer la información compartida) y sus preferencias en relación al horario de envío (mayor probabilidad que los mensajes sean leídos y tomados en cuenta). Otra fortaleza es la inclusión de la mayoría de estudiantes de primer año. Los resultados serían representativos para esta población, pudiendo ser extrapolables en otras facultades con características similares.

Las limitaciones del estudio también deben ser mencionadas. Primero, el contenido de los SMS no fue revisado por un experto en el tema. Sin embargo, es probable que ello haya tenido un bajo impacto, pues para la confección de los SMS se utilizó material del curso. Segundo, el contenido de los SMS no fue validado. Es posible que los mensajes no hayan sido totalmente entendidos por los participantes. No obstante, lo consideramos poco probable ya que se incluyó en el contenido la perspectiva de otros alumnos. Finalmente, es probable que utilizar la calificación final como único indicador de aprendizaje sea una limitación. Sin embargo, dado que el componente cognitivo fue evaluado en tres de los cuatro rubros de evaluación, consideramos que la intervención pudo tener influencia en el desempeño general de cada participante, y así en el aprendizaje de la materia.

En conclusión, el uso de SMS para mejorar el rendimiento académico de estudiantes del primer año de medicina en un curso de Primeros Auxilios fue positivo. Los SMS no solo deberían contener información teórica.

Agradecimientos: al Sr. Renato Mario Velit por su apoyo durante la primera fase del estudio, al igual que a todos los participantes por su colaboración.

Contribuciones de autoría: Los tres autores concibieron la idea de investigación. RMCL y SBSY desarrollaron el proyecto, hicieron la recolección de los datos y obtuvieron el financiamiento. RMCL y JEPL realizaron el análisis estadístico. RMCL, SBSY y JEPL interpretaron los resultados. RMCL desarrolló la primera versión del manuscrito, a la cual SBSY y JEP colaboraron activa y críticamente. Los tres autores aprobaron la versión del manuscrito enviada.

Fuentes de financiamiento: el proyecto recibió financiamiento de la Sociedad Científica Médico Estudiantil Peruana (SOCIMEP) y del grupo CTO.

Conflictos de interés: los autores declaran no tener conflictos de interés. La institución financiadora no participó en ninguna fase del estudio.

\section{REFERENCIAS BIBLIOGRÁFICAS}

1. Curioso W, Carnero AM. Promoviendo la investigación en salud con Twitter. Rev Med Hered. 2011 Jul;22(3):12130.

2. Pander T, Pinilla $S$, Dimitriadis $K$, Fischer MR. The use of Facebook in medical education--a literature review. GMS Z Med Ausbild. 2014 Aug 15;31(3):Doc33. doi: 10.3205/ zma000925.

3. Brunet $P$, Cuggia $M$, Le Beux P. Recording and podcasting of lectures for students of medical school. Stud Health Technol Inform. 2011;169:248-52.

4. Sahu M, Grover A, Joshi A. Role of mobile phone technology in health education in Asian and African countries: a systematic review. Int J Electron Healthc. 2014;7(4):269-86. doi: 10.1504/IJEH.2014.064327.

5. Patcai J. Polling the audience using text messaging--a tool for medical education. Med Teach. 2011;33(8):684-5.

6. Broom MA, Adamson GT, Draper LR. Text messaging in medical education. Pediatrics. 2014 Mar;133(3):e491-3. doi: 10.1542/peds.2013-1529.

7. Pimmer C, Linxen S, Gröhbiel U, Jha AK, Burg G. Mobile learning in resource-constrained environment: a case study of medical education. Med Teach. 2013 May;35(5):e1157-65. doi: 10.3109/0142159X.2012.733454.

8. Carpenter SK, Cepeda NJ, Rohrer D, Kang SHK, Pashler H. Using spacing to enhance diverse forms of learning: review of recent research and implications for instruction. Educ Psychol Rev. 2012 Sep 1;24(3):369-78.

9. Brown ON, O'Connor LE, Savaiano D. Mobile MyPlate: a pilot study using text messaging to provide nutrition education and promote better dietary choices in college students. J Am Coll Health. 2014;62(5):320-7. doi: $10.1080 / 07448481.2014 .899233$.

10. Kim SH, Mims C, Holmes KP. An introduction to Current Trends and Benefits of Mobile Wireless Technology Use in Higher Education. AACE Journal. 2006 Jan;14(1):77100.

11. Tiong-Thye G, Boon-Chong S, NianShing C. The impact of persuasive SMS on students's self-regulated learning. Brit J Educ Technol. 2012;43(4):624-40.

12. Busis N. Mobile phones to improve the practice of neurology. Neurol Clin. 2010 May;28(2):395-410. doi: 10.1016/j.ncl.2009.11.001.

13. Chang AY, Ghose S, Littman-Quinn R, Anolik RB, Kyer A, Mazhani L, et al. Use of movile learning by residents physicians in Botswana. Telemed J E Helath. 2012 Jan-Feb;18(1):11-3. doi: $10.1089 / \mathrm{tmj} .2011 .0050$.

14. Giunta A, Di Stefani A, Chimenti S. Mobile phones: a role in teaching dermatology? Dermatology. 2011;222(1):22-3. doi: 10.1159/000317074.

15. Richardson A, Littrell OM, Challman $S$, Stein P. Using text messaging in an undergraduate nursing course. J Nurs Educ. 2011 Feb;50(2):99-104. doi: 10.3928/01484834-20101230-04.

Correspondencia: Rodrigo M. Carrillo Larco Dirección: Av. La Merced 845 Dpto. 502, Surco.

Teléfono: +51987640892

Correo electrónico:rodrigo.carrillo@upch.pe 